\title{
Zur Begriffsbestimmung, Popularität und Kritik des Expressionismus in der kunsthistorischen Kommentarliteratur
}

\author{
Regine Prange
}

1.

Das revolutionäre Selbstverständnis der künstlerischen Bewegung des Expressionismus war eng verflochten mit einem Neuansatz der Disziplin Kunstgeschichte. 1907, zwei Jahre nach Gründung der Künstlergruppe Brücke, promovierte Wilhelm Worringer in Bern mit seiner Arbeit Abstraktion und Einfühlung, die als Programmschrift des Expressionismus wahrgenommen worden ist, obwohl sie sich vornehmlich mit der Gotik befasst, der Worringer, wie der nordischen Vorrenaissancekunst insgesamt, einen der romanischen Sinnenfreude entgegengesetzten kristallinischen Abstraktionsdrang zuordnet. ${ }^{1}$ In den Jahren vor dem Ersten Weltkrieg, der Gründungszeit der Münchner Künstlervereinigung Der Blaue Reiter, nahm der Wiener Kunsthistoriker Max Dvořák eine idealistische Neubewertung der venezianischen Malerei vor, die seine spätere Deutung Tintorettos als Vertreter eines "manieristischen Expressionismus « vorbereitete. ${ }^{2}$ Worringers Stilpsychologie wie Dvořáks Kunstgeschichte als Geistesgeschichte stützten sich wesentlich auf den Ausdruckswert der Form jenseits ihrer Abbildfunktionen. Der Expressionismus als eine gegen die - angeblich - indifferente, passiv-deskriptive Kunst des Impressionismus gerichtete Bewegung trifft sich mit dem Aufbruch der Kunstgeschichte in eine ikonologische Deutungsperspektive, die sich als Korrektiv einer sindifferenten< positivistischen Tatsachenforschung und formalistischen Stilgeschichte versteht.

Daraus schon ist erklärlich, dass von einer neutralen analytischen Kommentarliteratur zum Expressionismus nicht die Rede sein kann, zumindest solange nicht wie die theoretischen Prämissen der Kunstgeschichte selbst in den Fokus rückten. Die vergangene Kunst wurde im Licht der Gegenwart gedeutet, während

1 Wilhelm Worringer: Abstraktion und Einfühlung. Ein Beitrag zur Stilpsychologie. München 1908, 14. Aufl. 1987. Zum Verhältnis von Worringers Kunsthistoriographie zur Kunst des Expressionismus s. Magdalena Bushart: Der Geist der Gotik und die expressionistische Kunst. München 1990; Claudia Öhlschläger: Abstraktionsdrang. Wilhelm Worringer und der Geist der Moderne. München 2005. Zum Topos des Kristalls s. Regine Prange: Das Kristalline als Kunstsymbol. Paul Klee und Bruno Taut. Zur Theorie des Abstrakten in Kunst und Kunsttheorie der Moderne. Hildesheim u. a. 1991.

2 Hans Aurenhammer: "Max Dvořak, Tintoretto und die Moderne: Kunstgeschichte, ,Vom Standpunkt unserer Kunstentwicklung « betrachtet«, in: Wiener Jahrbuch für Kunstgeschichte IL (1997), S. 9-294, hier S. 28. 
man die Kunst der Gegenwart durch ihre Projektion in die Vergangenheit legitimierte. Ein Kokoschka fand sein historisches Pendant nicht nur in Tintoretto, ${ }^{3}$ sondern auch in Grünewald, wie zum Beispiel einer unvollendeten, postum veröffentlichten Schrift des Münchner Kunsthistorikers Fritz Burger zu entnehmen ist. Während des Kriegsdienstes in Nordfrankreich entworfen, wo Burger im Frühjahr 1916 fiel, endet diese Skizze einer universalen Kunstgeschichte mit dem Ergebnis, dass "Deutschland das klassische Land des >Expressionismus« sei. $^{4}$ Unter Hinweis nicht etwa auf zeitgenössische Werke, sondern auf mittelalterliche und altdeutsche Beispiele heißt es: "Daher sein [des Deutschen] Ungeschick in der gefälligen repräsentativen Darbietung des Stoffes, daher die elementare Willenskraft, der heilige Ernst und die scheue Bescheidenheit seiner Werke. $\ll^{5}$ Auf Grund dieser Disposition entgeht der Deutsche dem modernen »Problem der Form «; Kunst bleibt »Offenbarung des Geistigen $[\ldots]$. $^{6}$

Mit dem Terminus >Expressionismus< wurde zunächst die sich vom Naturvorbild lösende, genuin französische, Malerei des Postimpressionismus bezeichnet. In diesem Sinne spricht Wilhelm Worringer 1911 von den »jungpariser Synthetisten und Expressionisten «. ${ }^{7}$ Noch Paul Fechter versucht 1914 den Kubismus als »Variante des Expressionismus « einzuordnen. ${ }^{8}$ Populär wurde der Begriff eigentlich erst im Nachhinein, als in den 20er Jahren Wilhelm Hausenstein und Worringer Krise und Ende des Expressionismus verkündeten und Franz Roh den >NachExpressionismus ausrief. ${ }^{9}$ Auch wenn ein Teil der expressionistischen Künstler

3 Hans Aurenhammer: »Max Dvořak über Oskar Kokoschka: eine handschriftliche Fassung des Vorworts zu >Variationen über ein Thema «, in: Oskar Kokoschka-aktuelle Perspektiven. Hg. Hochschule für angewandte Kunst in Wien. Wien 1998, S. 34-40, bes. S. 35 .

4 Fritz Burger: Weltanschauungsprobleme und Lebenssysteme in der Kunst der Vergangenheit. München 1918, S. 86.

5 Ebd. Den zitierten Zeilen sind u. a. Abbildungen von Skulpturen des Bamberger Doms, des Frankfurter Hexenbildes von Baldung Grien, von Grünewalds Isenheimer Altar und einer Zeichnung Dürers zugeordnet.

6 Ebd., S. 87.

7 Wilhelm Worringer: „Entwicklungsgeschichtliches zur modernsten Kunst «, in: Im Kampf um die Kunst (1911), S. 92-99, hier S. 93. Vgl. Magdalena Bushart: Der Geist der Gotik... (wie Anm. 1), S. 46 f.

8 Paul Fechter: Der Expressionismus. München 1914, 3. Aufl. 1919, S. 35. Vgl. dagegen Ludwig Coellen: Die Neue Malerei. München 2. Aufl. 1912. Hier wird die von Cézanne eingeleitete Abkehr vom Naturalismus insgesamt unter das Motto einer neuen Romantik gestellt. Den Kubismus Picassos weist Coellen, historisch korrekter als Fechter, als das dominante Vorbild der »Jüngsten, die sich Expressionisten nennen «, aus. (Ebd., S. 67, Hervorhebung von L.C.)

9 Wilhelm Hausenstein: Die Kunst in diesem Augenblick. München 1920. Wiederabdruck von Auszügen in: W.H.: Die Kunst in diesem Augenblick. Aufsätze und Tagebuchblätter aus 50 Jahren. Ansbach 1960, S. 262-271 sowie S. 279-283. Wilhelm Wor- 
sich 1918 noch für eine sozialistische Umformung der Gesellschaft engagierte, schien einige Jahre später festzustehen, dass sich das anarchisch-kreative Ausdruckspotential der Bewegung durch eine bloß noch dekorative Verwendung expressionistischer Formen erschöpft hatte. »Längst ist Expressionismus kunstgewerblich ausgewalzt «, schreibt Hausenstein $1920 .{ }^{10}$ Die einst von ihm selbst geteilte Hoffnung auf eine große Stilsynthese im Sinne der auch von Coellen beschworenen »Fleischwerdung des überindividuellen Kulturgeistes« war dahin. ${ }^{11}$

Um nun, wie hier projektiert, zu einer Deutung der Deutungsgeschichte des Expressionismus zu gelangen, bedarf es einer Perspektive, die nicht nur deskriptiv die Begriffe und ihre Wandlung nachverfolgt, sondern in den Entwicklungen und Verwerfungen des Diskurses das zugrundeliegende Problem der Repräsentation reflektiert sieht. In diesem Sinne wäre, was hier nur skizzenhaft geschehen kann, das fundamentale Postulat des Ausdrucks zu fokussieren, das die Theorie des Expressionismus in zentralen Topoi variiert und verdichtet. Wie das Beispiel Burgers deutlich macht, bezieht sich Ausdruck in der expressionistischen Leseweise nicht auf die Äußerung eines alltäglichen kontingenten Gefühls oder Gedankens, sondern auf einen letzten, nicht mehr hintergehbaren Ursprung und ist insofern ein religiöses Gedankenbild. Seine Verifizierung wird geleistet durch die anachronistische Parallelisierung der Gegenwartskunst mit der Kultur des christlichen Mittelalters, aber auch durch den Rekurs auf vorästhetische sogenannte >primitive< Kunst überhaupt. ${ }^{12}$

Der Terminus >Ausdruck < postuliert eine unmittelbar gestaltete Übereinstimmung eines inneren seelischen Gehalts mit einer Form. Er ist, was angesichts der Gotik-Begeisterung der Expressionisten verwundern mag, erst im Zeitalter des bürgerlichen Neoklassizismus, durch Winckelmann nämlich, in die Kunsthistoriografie eingeführt worden: ${ }^{13}$ Das Schöne wird durch den Ausdruck erst au-

ringer: Künstlerische Zeitfragen. Lesung vor der Deutschen Goethegesellschaft, München, Nov. 1920. München 1921. Franz Roh: Nachexpressionismus. Magischer Realismus. Probleme der neuesten europäischen Malerei. Leipzig 1925.

10 Wilhelm Hausenstein: Die Kunst... (wie Anm. 9), S. 283.

11 Ludwig Coellen: Die neue Malerei (wie Anm. 8), S. 67.

12 S. u. a. die Illustrierungen folgender Bände: Klaus Lankheit (Hg.): Der Blaue Reiter. Hg. von Wassily Kandinsky und Franz Marc (1912). Dokumentarische Neuausgabe. München 1967; Hermann Bahr: Expressionismus. München 1916; Fritz Burger: Weltanschauungsprobleme... (wie Anm. 4).

13 Johann Joachim Winckelmann: Geschichte der Kunst des Alterthums. Hg. v. Heinrich Meyer/Johann Schulze. Bd. 2. Dresden 1811, Buch 4, Kapitel 2, § 24: [...] so kann die reine Schönheit allein nicht der einzige Vorwurf unserer Betrachtung seyn, sondern wir müssen dieselbe auch in den Stand der Handlung und der Leidenschaft setzen, welches wir in der Kunst mit dem Worte Ausdruck begreifen.« (Hervorhebung von J.J.W.) S.a. die weiteren Ausführungen in Buch 5, Kap. 2 und 3. 
thentisch, wenngleich dieser sich nicht verselbständigen darf, denn dann deformiert er das Schöne. Das moderne Dilemma der Repräsentation zeigt sich schon hier. Zum einen definierte die (auch Winckelmann inspirierende) Wissenschaft der Ästhetik das gebildete und bildsame bürgerliche Individuum durch das aus feudalen und klerikalen Zwängen entbundene subjektive Vermögen zum >Ausdruck r. Zum andern konnte dessen übergeordnete Geltungsmacht nur durch Vereinbarung mit einer objektiven Autorität beglaubigt werden. Deren Quelle jedoch kann nur durch Setzung bestimmt werden, etwa durch die Postulierung eines Kollektiv-Subjekts des Geschmacksurteils nach Kant oder durch die Prämisse einer sinneren Notwendigkeit in der Kunstlehre Kandinskys. ${ }^{14}$ Somit wurzelt in der Kategorie des Ausdrucks, die sowohl das geisteswissenschaftliche Projekt der Bedeutungsforschung als auch die expressionistische Kunst anleitet, das Legitimitätsproblem der künstlerischen Moderne schlechthin. Nachdem der Naturalismus des 19. Jahrhunderts unwiderruflich die antike Norm des Schönen verworfen hatte, war die objektive Größe des Subjektiven nur noch in diesem Subjektiven selbst lokalisierbar; die Psychologie wurde Leitwissenschaft auch der ästhetischen Theorie. Konrad Fiedler lieferte auf dieser Grundlage einen Lösungsvorschlag. Sein Konzept einer künstlerischen Ausdrucksbewegung schien das Unvereinbare vereinbaren, empirische Erfahrung und autonome Schöpfung synthetisieren zu können. ${ }^{15}$ Es wurde daher zum Generalschlüssel der postimpressionistischen Kunst und ihrer Kommentierung. Wenn Fechter »den Boden für ein neues seelisch metaphysisches Formwerden « im frühen 19. Jahrhundert bereitet sieht, das der Expressionismus realisiere, lässt sich dies - auch im Sinne Fiedlers - als eine psychologische Reformulierung des platonischen Idea-Konzepts lesen. ${ }^{16}$ Das nach wie vor klassizistische Fundament erschließt sich aus der Kritik am dekorativen Stilisieren der symbolistischen Generation: Erst die Losung »Zurück zum Gefühl!«, die die Abkehr von der Natur also durch eine innere Natur ergänzt, kann »wieder zur Kunst als Lebensausdruck « führen. ${ }^{17}$

Eben diese Implantierung eines essentiell-humanen Gehalts, der die Allgemeingültigkeit der expressionistischen Gestaltung verbürgen sollte, scheiterte. Der Expressionismus habe nach allem anderen auch sich selbst gesprengt »in dem

14 Zur Konstruktion der Allgemeingültigkeit des Geschmacksurteils bei Kant s. Regine Prange: Die Geburt der Kunstgeschichte. Philosophische Ästhetik und Empirische Wissenschaft. Köln 2004. Sein Ideal der inneren Notwendigkeit entwickelt Kandinsky u. a. in seinem Aufsatz »Über die Formfrage«, in: Der Blaue Reiter (wie Anm. 12), S. $132-188$.

15 Konrad Fiedler: »Über den Ursprung der künstlerischen Tätigkeit « (1881), in: K.F.: Schriften zur Kunst I. Hg. von Gottfried Boehm, 2. Aufl. 1991.

16 Paul Fechter: Expressionismus (wie Anm. 8), S. 6.

17 Ebd., S. 21. 
Augenblick, wo Kunst und Künstler sich vermaßen, das Metaphysische unmittelbar ergreifen zu wollen. ${ }^{18}$

\section{2.}

Die im Ersten Weltkrieg mit politisch imperialen Zielen konforme Intention, durch die expressionistische Kunst »europäische Geltung « zu erlangen, ja die »Gestaltungskraft der französischen Rasse « aufzunehmen, um, wie einst die angeblich deutsche Gotik, »die fernsten Interessensgebiete der Erde dem deutschen Gedanken « zu gewinnen, ${ }^{19}$ zerbrach schon an der Realität des Ersten Weltkriegs, dessen Materialschlachten der von zahlreichen Künstlern und Kunsthistorikern geteilten Begeisterung für den Kampf um das geistige Erbe der Nation den Boden entzog. Vollends vom internationalen Kunstfeld abgeschnitten wurde Deutschland schließlich durch die nationalsozialistische Kulturpolitik. Für viele der aus ihren Ämtern Vertriebenen oder mit Malverbot Belegten war die Ächtung des Expressionismus ein Rätsel, denn sie verstanden sich, schon durch den Bezug zur Gotik, als Bewahrer einer authentisch vaterländischen Tradition. ${ }^{20}$

Die Situation nach dem Zweiten Weltkrieg kann nicht mehr von Deutschland aus betrachtet werden, das durch Hitlers Vertreibung der intellektuellen Eliten, zu denen auch zahlreiche expressionistische Künstler gehörten, zur kulturellen Provinz gemindert war, weder politisch noch kulturell handlungsfähig. Als neues Zentrum der Kunstavantgarde hat sich schon in den 30er und 40er Jahren New York etabliert, nicht zuletzt auf der Basis des regen Einflusses europäischer Emigrantenzirkel. Alfred Barrs Konstruktion der Avantgarde-Geschichte im Rahmen seiner 1936 organisierten Ausstellung >Cubism and Abstract Art $<$ markiert eine Historisierung, ja Marginalisierung des deutschen Expressionismus im Konzert der Ismen. In einem das Cover des Ausstellungskatalogs schmückenden Schaubild, das die Genealogie zweier Formen abstrakter Kunst, der »Non-Geometrical Abstract Art « und der »Geometrical Abstract Art «, dokumentiert, wird unter der Bezeichnung »Abstract Expressionism« nur der Künstlergruppe Der Blaue Reiter ein relevanter Traditionsort zugewiesen. Barr notiert, dass der von Kandinsky, Klee und Marc entwickelte abstrakte Expressionismus in seiner Bedeutung gleich nach dem Kubismus rangiere. ${ }^{21}$

18 Wilhelm Hausenstein: Die Kunst... (wie Anm. 9), S. 271.

19 Karl Scheffler: Deutsche Kunst. Berlin 1915, S. 93 f. Zur nationalen Bestimmung des Expressionismus. Magdalena Bushart: Der Geist der Gotik... (wie Anm. 1), S. $110 \mathrm{ff}$.

20 S. z. B. Barlachs Klage, zitiert bei Bushart ebd., S. 227.

21 Alfred Barr: Cubism and Abstract Art. New York 1936, S. 64. Die Bezeichnung 'Abstrakter Expressionismus \& findet sich auch schon bei Eckart von Sydow: Die deutsche expressionistische Kultur und Malerei. Berlin 1920, S. 105 ff., hier mit Bezug auf Alexander Kanoldt, Franz Marc und Karl Schmidt-Rottluff (!). Die »Epochen des abstrakten 
Der in New York lehrende, höchst einflussreiche Kunsthistoriker Meyer Schapiro hat Barrs formalistischen Stilbegriff kritisiert und zugleich den Rang Kandinskys für sein alternatives ausdruckstheoretisches Geschichtskonzept hervorgehoben, das er explizit an das künstlerische Selbstverständnis bindet. ${ }^{22}$ Doch selbst diese begrenzte Anerkennung des deutschen Expressionismus wird von einer anderen Autorität der New Yorker Szene vehement bestritten. Der Kunstkritiker Clement Greenberg konstatiert brüsk, dass die »Expressionisten noch nicht damit zu Ende gekommen sind, herauszuarbeiten, was van Gogh nur angedeutet hatte, und auch noch nicht alle Hinweise ausgeschöpft haben, die in Cézannes frühem Stil zu finden sind. $\aleph^{23}$ Mit anderen Worten: Die expressionistische Malerei fällt hinter die Errungenschaften der historischen Avantgarde der $1870 \mathrm{er}$ und 1880er Jahre zurück. Maßstab dieser geringen Achtung ist das für Greenberg obligate Spannungsverhältnis zwischen der physischen Flächigkeit des Bildes und seiner Raumillusion. Die von den Expressionisten nicht genügend beachtete Kernaufgabe avantgardistischer Methoden liegt demzufolge darin, dem Flächencharakter des Bildes Geltung zu verschaffen und gegenüber dieser maßgeblichen Selbstreferenz möglichst die Fremdreferenz auszuschalten. Das zentrale Problem, so Greenberg, besteht darin, »jeden Bestandteil der Tiefenillusion auf einen Flächenaufbau zu beziehen, der in ästhetischer Hinsicht mit der Tiefenillusion gleichberechtigt ist. $\ll^{24}$

In der jungen Bundesrepublik Deutschland ist die Rezeption des Expressionismus freilich durch eine ganz andere Interessenlage bestimmt. Hier geht es nicht, wie im New York der Abstrakten Expressionisten, um die Inthronisierung und Abgrenzung einer brandneuen, bereits zu internationalem Ruhm gelangten künstlerischen Bewegung, sondern um Wiederanschluss an die internationale Kunstszene. Der Expressionismus, verfemt unter Hitler, »repräsentierte das wahre, humanistisch und demokratisch eingestellte Deutschland, das vom Nationalsozialismus nur vorübergehend okkupiert worden war $[\ldots] . \ll^{25}$ Es galt an diese Tradition wieder anzuknüpfen, um den deutschen Anteil an der Tradition der Kunstmoderne zu demonstrieren. Aus dem Widerspruch zwischen bekennendem Internationalismus und dem Beharren auf einer nationalen Kunst ergibt sich das spezifische Dilemma der Expressionismus-Kommentierung, die sich in

Expressionismus in der bildenden Kunst « (ebd., S. 137) werden zudem, in Nachfolge Worringers, bis in die jüngere Steinzeit und ihre geometrische Ornamentik zurückverfolgt.

22 Meyer Schapiro: »Das Wesen der abstrakten Malerei« (1937), in: M.S.: Moderne Kunst. 19. und 20. Jahrhundert. Ausgewählte Aufsätze. Köln 1981, S. 209-237.

23 Clement Greenberg: „Cézanne und die Einheit der modernen Kunst«, in: C. G.: Die Essenz der Moderne. Ausgewählte Essays und Kritiken. Hg. Karlheinz Lüdeking. Dresden 1997, S. 174-189, hier S. 175.

24 Ebd., S. 183.

25 Magdalena Bushart: Der Geist der Gotik... (wie Anm. 1), S. 227. 
ihrer affirmativen Funktion wieder und weiterhin mit politischen Repräsentationsinteressen verbindet. Ausschlaggebend ist dabei die bejahende Referierung der expressionistischen Ursprungsmythologie, denn diese ist die Klammer, die das Unvereinbare vereint: das allgemein gültige Gesetz und die individuelle Eigenart und Selbständigkeit nationaler Kunstsprachen.

In diesem Sinne wurde die erste, überwiegend retrospektiv auf die internationale Tradition der Moderne ausgerichtete documenta, 1955 in den Ruinen des Kasseler Friederizianum veranstaltet, mit einem visuellen Vorwort ausgestattet, das ganz im Geiste der expressionistischen Tradition etwa des Almanachs Der Blaue Reiter, den >Ursprung< der Kunst aufruft: In der Eingangshalle traf der Besucher auf große Fototafeln, die exotische Werke aus Afrika und dem präkolumbianischen Mittelamerika, frühromanische Reliefs, etruskische Skulpturen u. a. abbildeten. Nach dieser »Einstimmung auf die Moderne durch das Archaische « empfing Wilhelm Lehmbrucks Plastik Kniende (1911) als erstes Kunstwerk die Besucher. ${ }^{26}$ Ein gesamter Raum war deutschen Expressionisten - darunter Franz Marc, August Macke, Ernst Ludwig Kirchner, Alexej Jawlensky, Emil Nolde, Schmidt-Rottluff, Otto Mueller, Max Pechstein und Gabriele Münter gewidmet. Ein Kabinett der Kandinsky-Bilder inszenierte siebzehn Werke auf schwarzem Grund. Das größte Bild der ganzen Ausstellung war allerdings ein zeitgenössisches, von Fritz Winter gemaltes. Dieser einstige Schüler Kandinskys und Klees am Bauhaus wurde durch die räumliche Positionierung seines Werks als Bindeglied zwischen den deutschen Informellen (Baumeister, Nay) und den Meistern des Fauvismus und Kubismus - Matisse und Picasso - eingesetzt, was gewissermaßen den Wunsch nach einem deutschen Pollock hervortreten lässt, einem nationalen Künstlerhelden, der den Vergleich mit den Heroen der französischen Avantgarde nicht zu scheuen braucht.

Der Kunsthistoriker Werner Haftmann stand dem Malerei-Professor Arnold Bode bei der Durchführung der Ausstellung entscheidend zur Seite. Er ist zudem Autor eines schon 1954 erstmals publizierten und seither immer wieder aufgelegten Standardwerks zur Malerei des 20. Jahrhunderts, in dem der auch die Ausstellung leitende Geschichtsentwurf ausgeführt ist. Anders als seine Fachkollegen vor dem Krieg hütet sich Haftmann davor, das Deutsche des deutschen Expressionismus und dessen Weltgeltung herauszukehren. Dieser Inhalt lässt sich gleichwohl in der Anlage des Buchs erkennen, das als Entwicklungsgeschichte vorgestellt wird, jedoch in jedem seiner Kapitel dem Expressionismus bzw. dem Expressiven Raum gibt und seine Präsenz somit in allen Facetten der Kunstmoderne aufzeigt. Haftmann beschwört einen ursprünglichen, überall gleichen Im-

26 Walter Grasskamp: »documenta - kunst des XX. jahrhunderts - internationale ausstellung im museum friderizianum in kassel. 15. Juli bis 18. September «, in: Die Kunst der Ausstellung. Eine Dokumentation dreißig exemplarischer Kunstausstellungen dieses Jahrhunderts. Hg. Bernd Klüser/Katharina Hegewisch. Frankfurt a. M./Leipzig 1991, S. $116-125$, Zitat S. 117. 
puls zur Kunst. Nach dem Krieg habe sich »das große Einverständnis, das die schöpferischen Kräfte Europas über alle trennenden Umstände hinweg miteinander verband «, zum ersten $\mathrm{Mal}$ in »aller Deutlichkeit « manifestiert. ${ }^{27}$ Der anschließende Kommentar zur New Yorker Schule offenbart das latente Argument, denn in dem Maß wie jene Malergruppe die »Mittel der abstrakten Malerei als Möglichkeit zu einer spontanen Niederschrift psychischer Regungen « einsetzte, bediente sie sich, so Haftmann, der »Idee von der >psychischen Improvisation<, die Klee und Kandinsky schon einmal vor dem Ersten Weltkrieg hatten aufklingen lassen « und die auch von den deutschen und französischen Informellen wiederum beglaubigt wurde. ${ }^{28}$

Wenden wir uns abschließend zwei bekannten Autoren zu, die dem deutschen Expressionismus ein eher negatives Zeugnis ausstellten. Berühmt-berüchtigt ist Hans Sedlmayrs Deutung der künstlerischen Moderne als einer Krankheitsgeschichte, die den Menschen von Gott entferne und damit der Kunst jeden wahrhaft ethischen Sinn raube. In seinem Buch Verlust der Mitte illustriert Kokoschkas Stilleben mit enthäutetem Hammel und Hyazinthe (1910) diesen vermeintlich pathologischen Kern, auch wenn Sedlmayr konzediert, dass das Bild rein »künstlerisch genommen $[\ldots]$ von seltener innerer Konsequenz und >Dichte « ${ }^{29}$ sei. Sein Vorwurf gilt auch nicht den dargestellten widrigen Dingen, sondern dem Umstand, dass das Bild »jede Beziehung auf das Menschliche verloren hat und nur das Schillernde sucht. Das Versenken in das Bild führt in das Reich des Morbiden und dies um so gefährlicher, als es mit Meisterschaft das Verführerische dieser Region ins Bild bringt, das Bezaubernde, den >tödlichen Duft «.« ${ }^{30}$

Sedlmayr gelangt immerhin, wenn auch begrenzt durch sein theokratisches Weltbild, zu einer Problematisierung und Erklärung der expressionistischen Suche nach dem Ursprünglichen. In seinem 1955 erschienenen Buch Die Revolution der modernen Kunst befindet er im Kapitel über den Expressionismus bündig: »Es ist nicht möglich, zu den Ursprüngen durch das Opfer der Vernunft und des Geistes zurückzukehren. Es ist nicht möglich Mythos und Religion wiederherzustellen. «Doch schließt er das Kapitel mit den anerkennenden Worten, dass »der Versuch [...] etwas Heroisches « habe. ${ }^{31}$ Im folgenden Abschnitt hinterfragt er Paul Klees berühmtes Statement von 1918: „Diesseitig bin ich gar nicht

27 Werner Haftmann: Malerei im 20. Jahrhundert. Eine Entwicklungsgeschichte. München 1954, 7. Durchges. Aufl. 1987, S. 424 f.

28 Ebd., S. 427.

29 Hans Sedlmayr: Verlust der Mitte. Die bildende Kunst des 19. und 20. Jahrhunderts als Symptom und Symbol ihrer Zeit. Salzburg 1948, S. 215.

30 Ebd. Haftmann hingegen, konzentriert auf die Idee der psychischen Improvisation, deutet das Bild als visionär-halluzinatorische Selbstporträtierung des Künstlers, dessen Werke generell Ausdruck eines »dichterischen Daseinsgefühls« seien. Werner Haftmann: Malerei... (wie Anm. 27), S. 173.

31 Hans Sedlmayr: Die Revolution der modernen Kunst. Köln 1985, S. 114. 
faßbar. Denn ich wohne so gut bei den Toten wie bei den Ungeborenen. Etwas näher am Herzen der Schöpfung als üblich - und doch noch lange nicht genug." Nur der Naive, so Sedlmayr, lebe nahe am Herzen der Schöpfung, weshalb Klee sich die Haltung des Naiven aneigne. Die »bewusste Naivetät«, typisches Merkmal expressionistischen Schaffens, »schlägt damit aber in ihr Gegenteil um: in höchstes Raffinement. $\ll^{32}$ Bewusste Naivität, Gegenstück zur »absichtliche[n] Verrücktheit der Surrealisten ${ }^{33},{ }^{3}$ führt auch - so Sedlmayr -, zur Rechtfertigung und Einlassung des Dämonischen, was nachträglich seine Bewertung des Kokoschka-Stilllebens aufhellen mag. Sein etwas schlichtes, aber in sich nicht ganz unplausibles Erklärungsmuster ist Folgendes: Der Verlust der einst religiös, durch den Glauben an ein absolutes Wesen besetzten Mitte wird - notwendigerweise, wie Sedlmayr mit Hilfe anthropologischer Lehrmeinungen betont - kompensiert durch die Konstruktion von Idolen, denen sich die Kunst nun ersatzweise unterwirft. Deren wichtigste vier seien die Kunst selbst, Wissenschaft und Geometrie, die Technik und schließlich das (vom Expressionismus wie vom Surrealismus verehrte) Irrationale. Da keines der Idole, so Sedlmayr, »die Angewiesenheit des Menschen auf das wahre Absolute « zu befriedigen vermag ${ }^{34}$ muss immer ein Idol das andere stürzen; daher die permanente Kipp-Bewegung der Avantgarde zwischen einander entgegengesetzten Idealen; daher auch die Popularität der schließlich alle Autoritäten verneinenden nihilistischen Position.

In der Kritik am expressionistischen Konzept einer unmittelbaren Ausdrucksgebärde und in der anthropologischen Grundvorstellung einer notwendigen, institutionell gesicherten Weltorientierung trifft sich Sedlmayr mit dem Philosophen Arnold Gehlen, der in seinem 1960 publizierten Buch Zeitbilder einen grundlegenden Beitrag zur Soziologie und Ästhetik der modernen Malerei geliefert hat. Gleichwohl stellt sich Gehlen, in seiner Kritik an der »expressionistischen Verwirrung «, ${ }^{35}$ auf den Boden der Fiedlerschen Theorie. Diese »nichtrealistische Philosophie, die aus der sichtbaren Erscheinung heraus denkt und zugleich den Reflexionsstandpunkt durchhält [ist] wie keine andere geeignet, die moderne Kunst in Gedanken zu fassen. ${ }^{36}$ Wo Sedlmayr noch Sympathie für den heroischen, notwendig scheiternden Versuch der Expressionisten bekundet, eine religiöse Mitte wiederherzustellen, attackiert Gehlen den »Neu-Primitivismus«, der zwischen 1905 und 1910 »die künstlerischen Überlieferungen von 600 Jahren zerrissen und abgestreift « habe ${ }^{37}$ Denn durch den Bruch mit allen Traditionen verfehlte der deutsche Expressionismus - so Gehlens Argument - die

32 Ebd., S. 115.

33 Ebd., S. 131.

34 Ebd., S. 118.

35 Arnold Gehlen: Zeit-Bilder. Zur Soziologie und Ästhetik der modernen Malerei. Frankfurt a. M./Bonn 1960, 2. Aufl. 1965, S. 62.

36 Ebd., S. 60.

37 Ebd., S. 144. 
seit dem Impressionismus etablierte Reflexivität der Kunst, während sie in der französischen Malerei aufrechterhalten werde. Für die expressionistische »Selbstprogrammierung des Emotionalen $\aleph^{38}$ hat er nur beißenden Spott übrig, auch wenn er der »neue[n] Natürlichkeit« zugesteht, Widerstand gegen die Zwänge der Industriegesellschaft leisten zu wollen. »Die Affekte und Triebe drangen, in Wolken bunter Ideen gehüllt, nach oben, [...] an allen Enden regten sich Emanzipationen und sinnere Notwendigkeiten $<. \ll{ }^{39}$ Gehlens Leitidee ist zwar die der »Kommentarbedürftigkeit «moderner Kunst, doch das pseudo-archaische Vokabular der Expressionisten erfüllt die dem Kommentar zugewiesene Funktion, den orientierenden Halt der zerbrochenen Traditionen zu ersetzen, nicht. Hierauf nämlich zielt Gehlens anthropologische Entlastungstheorie, die im Gegensatz zu Freuds Lehre von einer notwendigen äußeren Triebmodellierung durch Tradition und Institution ausgeht. Im deutschen Expressionismus gelingt mithin nach Gehlens Auffassung die Konventionalisierung des künstlerischen Regelbruchs nicht; »die entformte Emotionalität [bleibt] amorph und qualitätlos $[\ldots] \ll{ }^{40}$ Die $\gg$ Operationsbasis des $>$ Erlebens $<$ ist als solche eben zu eng und instabil[...]. $\ll^{41}$ Erst der pictor doctus Paul Klee, den Gehlen vom genuinen Expressionismus absondert und auch Kandinsky vorzieht, erreicht wieder das Ideal einer peinture conceptuelle, die dem modernen Status des Bildes als einer "Reizfläche eigenen Rechts " genügt sowie optische und begriffliche Rationalität verbindet. ${ }^{42}$ Allerdings, und hier liegt das Problem von Gehlens Herangehensweise, belegt er dies vornehmlich anhand der elaborierten Eigenkommentare und Kunstlehren des Künstlers und nicht durch ihre strenge Abgleichung mit einer Werkanalyse. So wird der Künstlerkommentar, ein Merkmal auch der genuin kunsthistorischen Literatur jener Zeit, zur Theorie des Werks. Nur durch diese Überhöhung des Kommentars war die Einheitsidee Fiedlers einlösbar. Gehlen hat jedoch durch seine Problematisierung des Kommentars und die (an Greenbergs oben zitierten Einwand erinnernde) Stellungnahme zur künstlerischen Qualität expressionistischer Malerei Zugänge eröffnet, die von der historisierenden Kunsthistoriografie der zeitgenössischen Fachvertreter innovativ abweichen. Die weitere Entwicklung gab seiner Vermutung recht, dass solche Kritik »keine Zustimmung finden « würde, da ihr die geballten Interessen von Sammlern, Autoren und Museumsleitern entgegenstünden. ${ }^{43}$

Prof. Dr. Regine Prange, E-Mail: r.prange@kunst.uni-frankfurt.de

38 Ebd., S. 147.

39 Ebd., S. 133.

40 Ebd., S. 134.

41 Ebd., S. 136.

42 Ebd., S. 64. Zu Paul Klee als »pictor doctus« s. S. 102.

43 Ebd., S. 137. 